\title{
Feature Extraction for Patch-based Classification of Multispectral Earth Observation Images
}

\author{
Florin-Andrei Georgescu, Student Member, IEEE, Corina Vaduva, Member, IEEE, Dan Raducanu, \\ and Mihai Datcu, Fellow, IEEE
}

\begin{abstract}
Recently, various patch based approaches have emerged for high and very high resolution (VHR) multi-spectral images classification and indexing. This comes as a consequence of the most important particularity of multi-spectral data: objects are represented using several spectral bands that equally influence the classification process. In this paper, by using a patch based approach we are aiming at extracting descriptors that capture both spectral and structural information. Using both the raw texture data and the high spectral resolution provided by the latest sensors, we propose enhanced image descriptors based on Gabor, Spectral Histograms, Spectral Indices and a bag-of-words (BoW) framework. This approach leads to a scene classification that outperforms the results obtained when employing the initial image features. Experimental results have been performed on a WorldView2 scene and also on a test collection of tiles created using Sentinel 2 data. A detailed assessment of speed and precision was provided in comparison with state of the art techniques. The broad applicability is guaranteed, as the performances obtained for the two selected datasets are comparable, facilitating the exploration of previous and newly lunched satellite mission.
\end{abstract}

Keywords-BoW, Feature extraction, Gabor filters, Image Classification, Spectral features

\section{INTRODUCTION}

C ONSIDERING the exponential growth of the EO image data collections obtained from satellite and aerial sensors, in the last decades a lot of effort has been made for scene understanding and analysis.

Most of the image analysis approaches use feature extraction techniques and classification algorithms to automatically group input data similarities. Also, being generally inspired from the human visual system specialized in detecting specific image properties such as texture, color and shape, these methods usually require human-based data annotation, either for the training or the validation process [1].

Even though part of the effort made in EO data classification and understanding is based on multimedia image processing techniques there are attempts of using statistical text modeling approaches, such as author-topic-model [27] and author-genretopic-model [26]. These methods are using latent Dirichlet allocation to treat the topic mixture parameters as variables drawn from a Dirichlet distribution [27]. Also, [7], [8] and [9] present new techniques based on libraries of pretrained part detectors used for midlevel visual elements discovery in VHR remote sensing images. However, a lot of effort has been made to develop better texture, color and shape feature extraction techniques for both pixel and patch-based multispectral image analysis.
Widely used for image interpretation, segmentation, classification or change detection, the texture component can be defined as an arrangement of pixels as well as the spatial dependency between them in image [18]. Even though there are a lot of implementations, most of the texture analysis applications use techniques based on gray level co-occurrence matrix [10], wavelet transforms [21], Gauss-Markov random fields [16] and Gabor filtering [29].

By using only spectral information, color features are very easy to compute in comparison with texture and shape features, and are used on a large scale in scene classification and content based image retrieval applications. Some of the color features mostly used in remote sensing image analysis are color histograms [20] and color moments [24].

Local feature descriptors are another very important category of features. Like most of the available feature extraction methods for EO data, these local discriminative features have firstly been developed for multimedia image processing. The most popular and widely used techniques are referred to scale invariant feature transform [13], speeded up robust feature detector [2], rotation invariant feature transform [12], census transform histogram [28] and local binary pattern [17].

Currently evolving texture analysis and local feature extraction techniques have led the way to the BoW method. Even though BoW was initially used for video search, a lot of derivate methods that emerged from it could solve problems like image classification, image retrieval and object recognition. In the remote sensing community this technique has been recently introduced for image annotation, object classification, target detection and land use classification, and it has already proven its discrimination power in image classification. [3] In the BoW framework, there are several ways to generate the visual codebook. K-means is the most common clustering procedure, however in [20] there are some attempts in using random dictionaries [3].

It is known that in the case of multispectral EO image processing, objects are represented using several spectral bands that equally influence the classification process. In this paper we present an evaluation of State of the Art texture and spectral feature extraction methods such as Gabor [11], Spectral Histogram [23] and methods based on pure spectral information in a patch based approach to demonstrate their usability in the case of multispectral EO image data analysis and understanding. Relying on these classic approaches, we introduce new feature extraction techniques that will depict both spectral and structural information. The proposed methods can provide similar accuracy and enhanced computational 


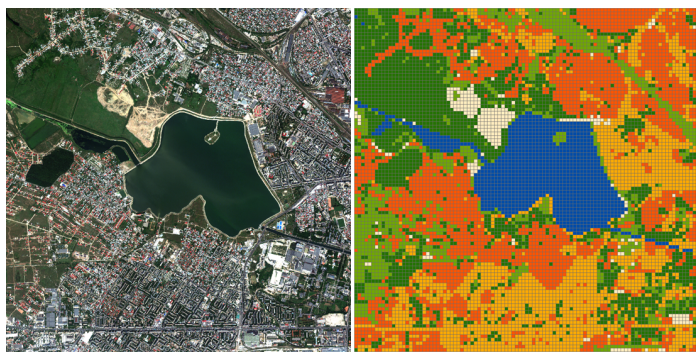

Fig. 1. WorldView-2 multispectral scene (Left). Reference annotation (Right)

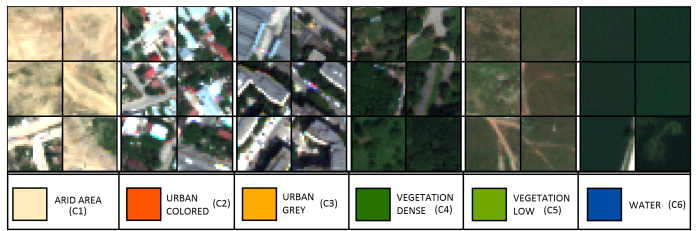

Fig. 2. Manual annotation legend and representative patches for each class. speed.

In order to provide relevant results we assessed the suggested methods in different scenarios, on a VHR WorldView2 (WV2) multispectral image and on a database of manually selected Sentinel-2 (S2) image patches. In the frame of performance evaluation, we assessed the proposed image descriptors in a comparative environment to demonstrate how texture and spectral descriptors can be combined together to obtain better classification results.

In Section 2 the main characteristics of the assessed feature extraction methods are presented, together with the algorithms to compute the feature vectors. Furthermore, in Section 3 we present the obtained results.

\section{FEATURE EXTRACTION}

In this section we briefly introduce how the image descriptors are computed. Our goal is to cover a wide range of feature extraction methods sensible to both structural and spectral information.

\section{A. Gabor features}

Based on a wavelet transform, multi scale Gabor filter is amongst the most used texture descriptors, being described in the MPEG-7 standard as a homogeneous texture descriptor (HTD) [23]. The Gabor representation has been proven to be optimal in the sense of minimizing the joint two-dimensional uncertainty in space and frequency [4], being well suited for texture detection and classification.

Different parameter setups are used for Gabor filter computation, but for best results, most of the authors use 2-6 frequencies and 2-8 orientations, as presented in [5], [6] and [19]. In our approach, we compute the Gabor filter bank for $\theta=6$ orientations and $\varphi=4$ scales. This means that we will have to filter each spectral band for every parameter combination. For each computed patch we extract the mean and standard deviation and keep them as Gabor features $(\mathrm{G})$. The size of a Gabor feature vector computed for a multispectral image patch with $n b$ number of bands will have the size $\theta \times \varphi \times n b \times 2$.

\section{B. Spectral Histogram features}

The Spectral Histogram is one of the most frequently used and basic descriptors that characterize the spectrum distribution in an image. Being inspired from the color histogram, we extend this terminology to the high spectral resolution of a multispectral image. A generic Spectral Histogram $(\mathrm{H})$ descriptor should be able to capture the spectral values distribution for image search and retrieval applications with resonable accuracy. [14]

For this simple, but efficient image descriptor, we compute for each band of a multispectral image patch the Spectral Histogram with $h b=64$ number of histogram bins for each of the $n b$ number of bands of the image, being highly motivated in dimensionality reduction of the feature vector [25]. The computed $n b$ histogram vectors are then merged together into the Spectral Histogram feature vector of size $h b \times n b$.

\section{Concatenated Gabor-Histogram features}

In order to improve the performances of the existing feature extraction methods, we propose joining Gabor features computed on the pure texture band with the Spectral Histogram features computed for all the spectral bands. We generate the texture band by using an average of the entire spectral bands available in the multispectral image.

Fig. 3, on the left side, shows the band correlations between two of the visible bands while the right side illustrates the correlation between a visible and an infra-red band of a WorldView-2 multispectral image. As we can easily observe, the visible bands are highly correlated with each other, and strongly uncorrelated with the infra-red bands. This may suggest that using the infra-red information to compute the image features will help us develop more efficient image descriptors that can represent the analyzed regions more accurately.

This new feature extraction method, based on both Gabor and Spectral Histogram features $(\mathrm{GH})$, will compute for a multispectral image patch a feature vector with the size of $2 \times \theta \times \varphi+h b \times n b$. elements, where $\theta$ represents the number of orientations and $\varphi$ the number of frequencies of the Gabor filter. The parameters $n b$ and $h b$ represent the number of bands in the multispectral image and the number of bins for each of the computed histograms respectively.

\section{Spectral Indices}

Using all the multispectral attributes, the spectral indices are a special category of image features that can be applied on multispectral images only. Taking into account the radiance values for each band and all the possible $(b 1-b 2) /(b 1+b 2)$ band

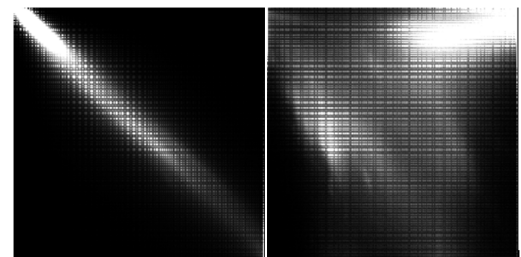

Fig. 3. Correlated (costal-blue vs. blue band - left) and Uncorrelated spectral bands (coastal-blue vs. infrared-2 - right) of a WorldView-2 image. 
ratios, where $b 1$ and $b 2$ refer to different band combinations, the number of computed spectral attributes is $n b(n b+1) / 2$, considering $n b$ is the number of bands in a multispectral image, as described in [15]. This leads to a very fast and easy to compute feature descriptor for multispectral images.

For our patch-based feature extraction employing the Spectral Indices computation, we modified the descriptor to use the same number of features as the original one, presented in [15]. In our patch based approach, we compute the feature vector with the size $n b(n b+1) / 2$ to be the mean of all Spectral Indices values from within the analyzed patch.

\section{E. Bag-of-words framework}

In the BoW model is performed a vector quantization of the spectral descriptors in an image against a visual codebook. Depending on the features used for codebook generation, different classification results may be obtained.

For the BoW feature descriptors we assessed the Spectral Indices computed for each pixel as described in [15] and we also used the radiance values for each pixel, as described in [3] with a dictionary size of 100 words. The codebook was generated using k-Means clustering on $10 \%$ of the computed features. Naturally, the size of the feature vector for a patchbased BoW is equal with the number of distinct words generated.

\section{Evaluation AND Discussions}

The methods we present are used to determine the maximum amount of information a feature descriptor can extract from an 8 band multispectral WorldView-2 image of $2 \mathrm{~m}$ spatial resolution. The scene used for experiments covers a surface of $25 \mathrm{~km}^{2}$ from Bucharest, Romania and it can be observed in Fig. 1 (left side). We selected this scene because of the numerous classes that can be identified and analyzed. Also, of big importance was the abundance of multispectral information provided by the 8 bands WorldView- 2 data.

In all the cases we used the same size for patch analysis. Without affecting the classification results, for this study we minimized the patch size to have $25 \times 25$ pixels, so it can cover a surface of $2500 \mathrm{~m}^{2}$.

For the evaluation procedure, we made a manual annotation of the scene (Fig. 1 right side) in which we considered six generic classes such as Arid area (C1), Urban Colored (C2), Urban Grey (C3), Vegetation Dense (C4), Vegetation Low (C5) and Water (C6). Fig. 2 illustrates the legend used for the annotation map representation (Fig. 1 - right) and some samples of the annotated patches.

In order to create the manual annotation map, the labeling was made using the dominant class present in the patches. Even though the representation from Fig. 2 is done using the red, green and blue bands, we made our best efforts to annotate the most suitable class based on the information from all the available spectral bands simultaneously.

During classification step we used support vector machines (SVM) and k-Nearest Neighbors. For the SVM setup we used radial basis function (RBF) kernel that have the gamma coefficient $\gamma=3.0518 \times 10^{-4}$ and the regression parameter
$C=5$. In the case of k-NN classification we used $k=10$ neighbours. Also, the qualitative and quantitative evaluation was made by computing confusion matrixes using as reference the manual annotated map.

In order to reveal the feature extraction and classification performances, Fig. 5 illustrates the computation speeds achieved for computing $1000 \times 1000$ pixels in patches of $25 \times 25$ pixels. For this benchmark we also used a Sentinel-2 image, with 4 spectral bands and a WorldView- 2 scene, with 8 spectral bands. The system architecture used in the processing has 16 GB of RAM and an 8 core $2.4 \mathrm{GHz}$ CPU.

\section{A. Data Analysis}

In order to identify the most suitable feature extraction method that can be used for multispectral image analysis, we have considered two scenarios: one to determine the performances of texture and spectral feature descriptors and another to benchmark the feature extraction methods for patchbased classification of EO multispectral images.

In the context of the first scenario, for data analysis and understanding, we used texture and spectral features, such as Gabor and Spectral Histogram in order to evaluate the best performing method suitable for multispectral image classification.

For texture analysis we extracted Gabor features on the full texture band, computed from the mean of the spectral bands, and we extended the assessment for the multispectral image with the full band information.

In the second scenario of our evaluation we completed a benchmark of Gabor (G), Spectral Histogram (H), GaborHistogram (GH), Spectral Indices (SI), Bag of Words from Spectral Indices (BSI) and Bag of Words from Spectral Values (BSV) descriptors to determine the best multispectral feature extraction method suitable for multispectral image analysis.

\section{B. Evaluation Results}

We evaluated the performances of Gabor and Spectral Histogram image features in the first scenario. Having as a start point the texture measure done by computing the average texture band used for Gabor feature extraction, and continuing with Spectral Histogram computation of the multispectral image, we observed that the results of the classification can be improved. Also, the possibility of increasing performance of the texture or spectral descriptors alone can be easily observed from the band correlation images presented in Fig. 3. This has led to the development of a Gabor-Histogram feature

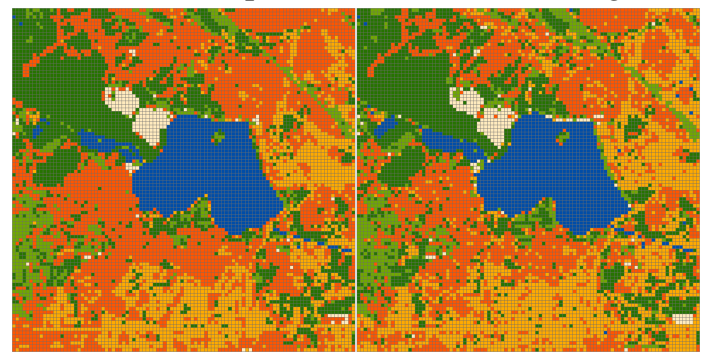

Fig. 4. k-NN classification of GH (left) vs. BSV (right) features 


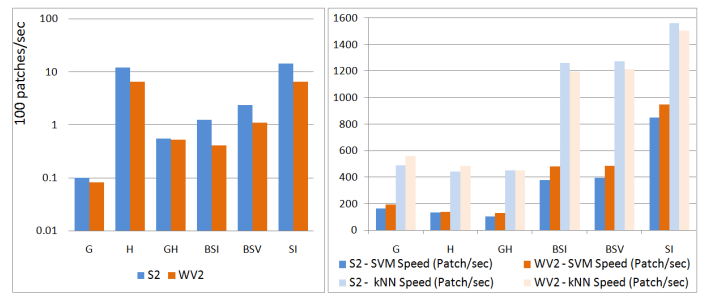

Fig. 5. Speed of feature extraction (Left) and feature classification (Right)

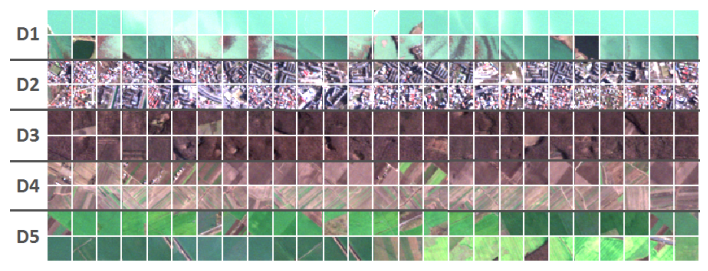

Fig. 6. Sentinel-2 database of thematic classes.

descriptor that computes the texture using Gabor method only for the average texture band and Spectral Histogram for all the multispectral bands.

Another reason for computing image features using GaborHistogram method instead of the classical ones is that this new method is faster than the classical Gabor feature descriptor approach computed for all the multispectral bands and has better classification accuracy. The same is also true for classical Histogram descriptor as it can be observed from Fig. 5 and Table. I.

From Table I, we can easily see that combining the two descriptors the classification using the new method increased the accuracy for SVM and k-NN classifications with up to $30 \%$ and $20 \%$ respectively in case of single band texture analysis and with up to 5\% for both SVM and k-NN when using all the multispectral bands for spectral histogram classification.

In the second scenario, we set up a benchmarking environment for the presented feature extraction methods in order to determine the best performing algorithm for multispectral image classification. In contrast to the previous case, in this new situation we will use the full power of the multiple spectral bands of the analyzed scene. Table II and III present the precision-recall (P-R) scores obtained for each of the assessed methods in the form of confusion matrixes for SVM and k-NN classification.

Even though the classification results are very tight for specific feature extraction methods and also for the same classifiers, we can observe from Table II and Table III, that for both SVM and k-NN classifications the BoW features have the highest average accuracy scores - BSI have an accuracy of $71.4 \%$ for SVM classification while BSV accuracy is $72.3 \%$ for $\mathrm{k}-\mathrm{NN}$. The BoW based features are followed by the proposed GH descriptor with an accuracy of $71.2 \%$ for SVM classification and $71.3 \%$ for k-NN classification.

A few initial setups have been made in the frame of the supervised classification. The training samples were randomly selected from the manual annotation file and the number of samples for each class represent $20 \%$ from the number of manually annotated classes.

Inspired from multimedia image classification, the values
TABLE I. K-NN VS SVM ACCURACY, WV2, SCENARIO 1

\begin{tabular}{|l|l|l|l|l|l|l|}
\hline & Gabor & \multicolumn{2}{|l|}{ Histogram } & \multicolumn{2}{|c|}{ Gabor-Histogram } \\
\cline { 2 - 7 } & SVM & k-NN & SVM & k-NN & SVM & k-NN \\
\hline C1 & $33.7 \%$ & $55.9 \%$ & $64.6 \%$ & $55.3 \%$ & $68.4 \%$ & $60.5 \%$ \\
\hline C2 & $70.1 \%$ & $83.5 \%$ & $68.8 \%$ & $88.7 \%$ & $71.8 \%$ & $89.7 \%$ \\
\hline C3 & $49.6 \%$ & $54.2 \%$ & $63.7 \%$ & $69.2 \%$ & $68.6 \%$ & $67.1 \%$ \\
\hline C4 & $46.4 \%$ & $68.3 \%$ & $69.8 \%$ & $73.8 \%$ & $72.7 \%$ & $74.8 \%$ \\
\hline C5 & $32.3 \%$ & $23.8 \%$ & $47.1 \%$ & $39.8 \%$ & $49.7 \%$ & $41.5 \%$ \\
\hline C6 & $93.0 \%$ & $93.3 \%$ & $95.4 \%$ & $94.1 \%$ & $96.0 \%$ & $94.4 \%$ \\
\hline
\end{tabular}

TABLE II. P-R ON SVM CLASSIFICATION, WV2, SCENARIO 2

\begin{tabular}{|l|l|l|l|l|l|l|l|l|}
\hline \multicolumn{2}{|c|}{} & $\mathrm{C} 1$ & $\mathrm{C} 2$ & $\mathrm{C} 3$ & $\mathrm{C} 4$ & $\mathrm{C} 5$ & $\mathrm{C} 6$ & Mean \\
\hline \multirow{2}{*}{$\mathrm{G}$} & $\mathrm{P}$ & $69.6 \%$ & $73.6 \%$ & $72.4 \%$ & $70.8 \%$ & $42.4 \%$ & $95.7 \%$ & $\mathbf{7 0 . 7} \%$ \\
\cline { 2 - 9 } & $\mathrm{R}$ & $80.1 \%$ & $71.6 \%$ & $65.0 \%$ & $73.4 \%$ & $53.7 \%$ & $96.8 \%$ & $73.4 \%$ \\
\hline \multirow{2}{*}{$\mathrm{H}$} & $\mathrm{P}$ & $64.6 \%$ & $68.8 \%$ & $63.7 \%$ & $69.8 \%$ & $47.1 \%$ & $95.3 \%$ & $\mathbf{6 8 . 2} \%$ \\
\cline { 2 - 9 } & $\mathrm{R}$ & $84.5 \%$ & $65.0 \%$ & $60.4 \%$ & $73.5 \%$ & $53.3 \%$ & $97.5 \%$ & $72.4 \%$ \\
\hline \multirow{2}{*}{$\mathrm{GH}$} & $\mathrm{P}$ & $68.4 \%$ & $71.8 \%$ & $68.6 \%$ & $72.7 \%$ & $49.7 \%$ & $96.0 \%$ & $\mathbf{7 1 . 2} \%$ \\
\cline { 2 - 9 } & $\mathrm{R}$ & $85.8 \%$ & $67.7 \%$ & $64.5 \%$ & $76.2 \%$ & $58.8 \%$ & $97.2 \%$ & $75.0 \%$ \\
\hline \multirow{2}{*}{ BSI } & $\mathrm{P}$ & $67.9 \%$ & $72.1 \%$ & $69.3 \%$ & $72.3 \%$ & $49.7 \%$ & $97.2 \%$ & $\mathbf{7 1 . 4} \%$ \\
\cline { 2 - 9 } & $\mathrm{R}$ & $83.8 \%$ & $70.9 \%$ & $65.6 \%$ & $76.0 \%$ & $59.7 \%$ & $96.5 \%$ & $75.4 \%$ \\
\hline \multirow{2}{*}{ BSV } & $\mathrm{P}$ & $65.2 \%$ & $70.2 \%$ & $70.3 \%$ & $66.4 \%$ & $52.0 \%$ & $95.9 \%$ & $\mathbf{7 0 . 0} \%$ \\
\cline { 2 - 8 } & $\mathrm{R}$ & $87.1 \%$ & $70.9 \%$ & $62.6 \%$ & $75.7 \%$ & $53.5 \%$ & $96.3 \%$ & $74.3 \%$ \\
\hline \multirow{2}{*}{ SI } & $\mathrm{P}$ & $55.6 \%$ & $68.6 \%$ & $57.8 \%$ & $58.2 \%$ & $40.6 \%$ & $94.5 \%$ & $\mathbf{6 2 . 5} \%$ \\
\cline { 2 - 8 } & $\mathrm{R}$ & $78.9 \%$ & $60.2 \%$ & $57.2 \%$ & $71.7 \%$ & $39.6 \%$ & $97.6 \%$ & $67.5 \%$ \\
\hline
\end{tabular}

TABLE III. P-R ON K-NN CLASSIFICATION, WV2, SCENARIO 2

\begin{tabular}{|l|l|l|l|l|l|l|l|l|}
\hline \multicolumn{2}{|c|}{} & $\mathrm{C} 1$ & $\mathrm{C} 2$ & $\mathrm{C} 3$ & $\mathrm{C} 4$ & $\mathrm{C} 5$ & $\mathrm{C} 6$ & Mean \\
\hline \multirow{2}{*}{$\mathrm{G}$} & $\mathrm{P}$ & $69.3 \%$ & $84.0 \%$ & $64.5 \%$ & $73.8 \%$ & $43.3 \%$ & $93.4 \%$ & $\mathbf{7 1 . 4} \%$ \\
\cline { 2 - 9 } & $\mathrm{R}$ & $71.4 \%$ & $68.9 \%$ & $70.5 \%$ & $76.2 \%$ & $60.7 \%$ & $97.5 \%$ & $74.2 \%$ \\
\hline \multirow{2}{*}{$\mathrm{H}$} & $\mathrm{P}$ & $55.3 \%$ & $88.7 \%$ & $69.2 \%$ & $73.8 \%$ & $39.8 \%$ & $94.1 \%$ & $\mathbf{7 0 . 1} \%$ \\
\cline { 2 - 9 } & $\mathrm{R}$ & $90.2 \%$ & $66.2 \%$ & $77.6 \%$ & $77.4 \%$ & $68.6 \%$ & $98.1 \%$ & $79.7 \%$ \\
\hline \multirow{2}{*}{$\mathrm{GH}$} & $\mathrm{P}$ & $60.5 \%$ & $89.7 \%$ & $67.1 \%$ & $74.8 \%$ & $41.5 \%$ & $94.4 \%$ & $\mathbf{7 1 . 3} \%$ \\
\cline { 2 - 9 } & $\mathrm{R}$ & $89.0 \%$ & $67.2 \%$ & $78.8 \%$ & $77.2 \%$ & $67.4 \%$ & $98.1 \%$ & $79.6 \%$ \\
\hline \multirow{2}{*}{ BSI } & $\mathrm{P}$ & $63.8 \%$ & $85.2 \%$ & $67.7 \%$ & $73.3 \%$ & $42.7 \%$ & $96.0 \%$ & $\mathbf{7 1 . 4} \%$ \\
\cline { 2 - 9 } & $\mathrm{R}$ & $83.2 \%$ & $67.5 \%$ & $73.5 \%$ & $77.5 \%$ & $65.0 \%$ & $96.4 \%$ & $77.2 \%$ \\
\hline \multirow{2}{*}{ BSV } & $\mathrm{P}$ & $64.3 \%$ & $78.0 \%$ & $74.2 \%$ & $76.7 \%$ & $44.5 \%$ & $96.0 \%$ & $\mathbf{7 2 . 3} \%$ \\
\cline { 2 - 9 } & $\mathrm{R}$ & $83.4 \%$ & $72.8 \%$ & $68.5 \%$ & $74.9 \%$ & $65.4 \%$ & $96.2 \%$ & $76.9 \%$ \\
\hline \multirow{2}{*}{ SI } & $\mathrm{P}$ & $64.1 \%$ & $80.4 \%$ & $70.2 \%$ & $74.6 \%$ & $37.6 \%$ & $94.1 \%$ & $\mathbf{7 0 . 2} \%$ \\
\cline { 2 - 9 } & $\mathrm{R}$ & $72.1 \%$ & $69.5 \%$ & $68.9 \%$ & $75.4 \%$ & $57.2 \%$ & $97.8 \%$ & $\mathbf{7 3 . 5 \%}$ \\
\hline
\end{tabular}

TABLE IV. P-R ON SVM CLASSIFICATION, S2

\begin{tabular}{|l|l|l|l|l|l|l|l|}
\hline \multicolumn{2}{|c|}{} & D1 & D2 & D3 & D4 & D5 & Mean \\
\hline \multirow{2}{*}{ G } & P & $98.53 \%$ & $97.08 \%$ & $98.61 \%$ & $98.61 \%$ & $98.76 \%$ & $\mathbf{9 8 . 3 2 \%}$ \\
\cline { 2 - 8 } & R & $98.82 \%$ & $97.58 \%$ & $97.87 \%$ & $98.78 \%$ & $98.62 \%$ & $98.33 \%$ \\
\hline \multirow{2}{*}{ H } & P & $97.88 \%$ & $97.02 \%$ & $96.34 \%$ & $96.66 \%$ & $97.44 \%$ & $\mathbf{9 7 . 0 7 \%}$ \\
\cline { 2 - 8 } & R & $98.96 \%$ & $93.15 \%$ & $97.05 \%$ & $97.97 \%$ & $98.51 \%$ & $97.13 \%$ \\
\hline \multirow{2}{*}{ GH } & P & $98.18 \%$ & $97.18 \%$ & $97.72 \%$ & $98.14 \%$ & $98.14 \%$ & $\mathbf{9 7 . 8 7 \%}$ \\
\cline { 2 - 8 } & R & $99.02 \%$ & $96.16 \%$ & $97.48 \%$ & $98.64 \%$ & $98.15 \%$ & $97.89 \%$ \\
\hline \multirow{2}{*}{ BSI } & P & $97.60 \%$ & $98.24 \%$ & $99.28 \%$ & $97.60 \%$ & $98.42 \%$ & $\mathbf{9 8 . 2 3 \%}$ \\
\cline { 2 - 8 } & R & $99.44 \%$ & $96.51 \%$ & $98.42 \%$ & $98.43 \%$ & $98.44 \%$ & $98.25 \%$ \\
\hline \multirow{2}{*}{ BSV } & P & $98.28 \%$ & $95.06 \%$ & $98.44 \%$ & $98.50 \%$ & $98.80 \%$ & $\mathbf{9 7 . 8 2 \%}$ \\
\cline { 2 - 8 } & R & $98.84 \%$ & $96.17 \%$ & $96.20 \%$ & $99.68 \%$ & $98.31 \%$ & $97.84 \%$ \\
\hline \multirow{2}{*}{ SI } & P & $98.56 \%$ & $96.00 \%$ & $99.38 \%$ & $95.68 \%$ & $98.38 \%$ & $\mathbf{9 7 . 6 0 \%}$ \\
\cline { 2 - 7 } & R & $99.18 \%$ & $95.14 \%$ & $99.12 \%$ & $96.02 \%$ & $98.69 \%$ & $97.63 \%$ \\
\hline
\end{tabular}

TABLE V. P-R ON K-NN CLASSIFICATION, S2

\begin{tabular}{|l|l|l|l|l|l|l|l|}
\hline \multicolumn{2}{|c|}{} & D1 & D2 & D3 & D4 & D5 & Mean \\
\hline \multirow{2}{*}{ G } & P & $96.14 \%$ & $93.92 \%$ & $98.20 \%$ & $99.00 \%$ & $93.38 \%$ & $\mathbf{9 6 . 1 3 \%}$ \\
\cline { 2 - 8 } & R & $98.71 \%$ & $92.98 \%$ & $93.17 \%$ & $97.14 \%$ & $99.17 \%$ & $96.23 \%$ \\
\hline \multirow{2}{*}{$\mathrm{H}$} & $\mathrm{P}$ & $96.04 \%$ & $96.72 \%$ & $96.14 \%$ & $98.40 \%$ & $94.80 \%$ & $\mathbf{9 6 . 4 2 \%}$ \\
\cline { 2 - 8 } & $\mathrm{R}$ & $99.44 \%$ & $92.85 \%$ & $95.61 \%$ & $95.63 \%$ & $99.04 \%$ & $96.52 \%$ \\
\hline \multirow{2}{*}{ GH } & $\mathrm{P}$ & $96.66 \%$ & $96.00 \%$ & $96.38 \%$ & $98.70 \%$ & $95.58 \%$ & $\mathbf{9 6 . 6 6 \%}$ \\
\cline { 2 - 8 } & $\mathrm{R}$ & $99.55 \%$ & $93.01 \%$ & $94.91 \%$ & $96.98 \%$ & $99.23 \%$ & $96.74 \%$ \\
\hline \multirow{2}{*}{ BSI } & $\mathrm{P}$ & $97.82 \%$ & $94.72 \%$ & $97.96 \%$ & $96.80 \%$ & $96.06 \%$ & $\mathbf{9 6 . 6 7 \%}$ \\
\cline { 2 - 8 } & $\mathrm{R}$ & $97.25 \%$ & $95.68 \%$ & $98.26 \%$ & $94.28 \%$ & $98.00 \%$ & $96.69 \%$ \\
\hline \multirow{2}{*}{ BSV } & $\mathrm{P}$ & $97.18 \%$ & $92.64 \%$ & $98.54 \%$ & $97.42 \%$ & $96.90 \%$ & $\mathbf{9 6 . 5 4 \%}$ \\
\cline { 2 - 7 } & $\mathrm{R}$ & $97.95 \%$ & $93.53 \%$ & $93.78 \%$ & $99.47 \%$ & $98.26 \%$ & $96.60 \%$ \\
\hline \multirow{2}{*}{ SI } & $\mathrm{P}$ & $97.90 \%$ & $96.18 \%$ & $99.14 \%$ & $95.32 \%$ & $95.24 \%$ & $\mathbf{9 6 . 7 6 \%}$ \\
\cline { 2 - 7 } & $\mathrm{R}$ & $98.71 \%$ & $92.87 \%$ & $98.26 \%$ & $94.67 \%$ & $99.61 \%$ & $96.82 \%$ \\
\hline
\end{tabular}

shown in the Tables II-V are obtained using the mean values of the P-R scores obtained by classifying the scene with 10 randomly generated training sets.

With the purpose to demonstrate the usability of feature 
extraction methods on various EO multispectral images, we also assessed the proposed methods on data from the recently released Sentinel-2 satellite. For this assessment, we use only 4 of the spectral bands provided by this satellite that has $10 \mathrm{~m}$ spatial resolution. With this setup we manually selected 2500 patches, of $25 \times 25$ pixels, grouped in five thematic classes of 500 patches each (D1 - Water, D2 - Urban, D3 - Forest, D4 - AgricultureLow, D5 - AgricultureHigh), presented in Fig. 6. High classification scores can be seen in Tables IV and $\mathrm{V}$, because of the patches uniformly distributed microtextures. Also, the purpose of this test database is to verify the image feature performances and not the classification accuracy.

\section{CONCLUSION}

Considering this paradigm of image understanding, the development of efficient feature extraction methods for multispectral data analysis and classification is not an easy task and no general approaches for efficient classification of satellite images are provided.

According to the experimental results, common texture and color descriptors can be adapted and successfully used for multispectral image analysis. Moreover, by combining texture and spectral features we can obtain more powerful descriptors. We also achieved in important results by using Spectral Indices descriptors, which are very fast and easy to compute.

Even though the most suitable image descriptors for multispectral image analysis prove to be the ones based on the BoW framework, the classical ones provide similar results with a shorter computation time. Moreover, the Gabor-Histogram descriptor, which computes both texture and spectral information leads to similar average accuracy rates.

The goal of this paper is to provide enhanced feature extraction methods that can be used on multispectral EO images for a better data understanding and classification.

\section{ACKNOWLEDGMENT}

The WorldView2 data was provided in the frame of the "2010 DigitalGlobe - 8 bands Research Challenge".

\section{REFERENCES}

[1] R. Bahmanyar et al., The Semantic Gap: An Exploration of User and Computer Perspectives in Earth Observation Images, IEEE Geos. Rem. Sens. Lett., vol. 12, no. 10, pp. 2046-2050, 2015

[2] H. Bay et al., Speeded-up robust features (SURF), Comput. Vis. Image Understand, vol. 110, no. 3, pp. 346-359, 2008

[3] S. Cui, G. Schwarz and M. Datcu, Remote Sensing Image Classification: No Features, No Clustering, IEEE J. Sel. Topics in Appl. in Earth Obs and Rem. Sens.,vol. 8, no. 11, pp. 5158-5170, 2015

[4] J. G. Daugman, Complete discrete 2D Gabor transforms by neural networks for image analysis and compression, IEEE Trans. Acoust., Speech, Signal Process., vol. 36, no. 7, pp. 1169-1179, 1988

[5] C. O. Dumitru et al., Information Content of Very High Resolution SAR Images: Semantics, Geospatial context and ontologies, IEEE J. Sel Topics in Appl. in Earth Obs and Rem. Sens., vol. 8, no. 4, pp. 16351650,2015

[6] C. O. Dumitru and M. Datcu, Information Content of Very High Resolution SAR Images: Study of Feature Extraction and Imaging Parameters, IEEE Trans. Geos. Rem. Sens., vol. 51, no. 8, pp. 4591-4610, 2013
[7] G. Cheng et al., Effective and Efficient Midlevel Visual ElementsOriented Land-Use Classification Using VHR Remote Sensing Images, IEEE Trans. Geos. Rem. Sens., vol. 53, no. 8, pp. 4238-4249, 2015

[8] G. Cheng et al., Multi-class geospatial object detection and geographic image classification based on collection of part detectors, IET Comput. Vis., vol. 9, no. 5, pp. 119-132, 2014

[9] G. Cheng et al., Auto-encoder-based shared mid-level visual dictionary learning for scene classification using very high resolution remote sensing images, IET Comput. Vis., vol. 9, no. 5, pp. 639-649, 2015

[10] R. M. Haralick et al., Textural features for image classication, IEEE Trans. Syst. Man. Cybern., vol. 3, no. 6, pp. 610-621, 1973

[11] S.E. Grigorescu et al., Comparison of texture features based on Gabor filters, IEEE Trans. Image Process., vol. 11, no. 10, pp. 1160-1167, 2002

[12] S. Lazebnik, C. Schmid and J. Ponce, A sparse texture representation using local affine regions, IEEE Trans. Pattern Anal. Mach. Intell., vol. 27, no. 8, pp. 1265-1278, 2005

[13] D. G. Lowe, Distinctive image features from scale-invariant keypoints, Int. J. Comput. Vis., vol. 60, no. 2, pp. 91-110, 2004

[14] B. S. Manjunath et al., Color and Texture Descriptors, IEEE Trans. Circuits Syst. Video Technol., vol. 11, no. 6, pp. 703-715, 2001

[15] G. Marchisio, F. Pacifici and C. Padwick, On The Relative Predictive Value Of The New Spectral Bands In The WorldView-2 Sensor, IEEE Symp. Geos. Rem. Sens., pp. 2723-2726, 2010

[16] R.D. Navarro, J.C. Magadia and E.C. Paringit, Estimating the GaussMarkov Random Field parameters for remote sensing image textures, TENCON, IEEE Region 10 Conf., pp. 1-6, 2009

[17] T. Ojala et al., Multiresolution gray-scale and rotation invariant texture classication with local binary patterns, IEEE Trans. Pattern Anal. Mach. Intell., vol. 24, no. 7, pp. 971-987, 2002

[18] M.T. Pham et al., Pointwise Graph-Based Local Texture Characterization for Very High Resolution Multispectral Image Classification, IEEE J. Sel. Topics in Appl. in Earth Obs and Rem. Sens., vol. 8, no. 5, pp. 1962-1973, 2015

[19] R. Roslan and N. Jamil, Texture feature extraction using 2-D gabor filters, IEEE Symp. on Comput. Applicat. and Ind. Electron., pp. 173178,2012

[20] K.E.A. van de Sande et al., Evaluationg Color Descriptors for Object and Scene Recognition, IEEE Trans. Pattern Anal. Mach. Intell., vol. 32, no. 9 , pp. 1582-1596, 2009

[21] P. Scheunders et al., Wavelet-based texture analysis, Int. J. Comput. Sci. Inf. Manage., vol.1, pp. 22-34, 1998

[22] S.F. Chang et al., Overivew of the MPEG-7 standard, IEEE Trans. Circuits Syst. Video Technol., vol. 11, no. 6, pp. 688-695, 2001

[23] T. Sikora, The MPEG-7 Visual Standard for Content DescriptionAn Overview, IEEE Trans. Circuits Syst. Video Technol., vol. 11, no. 6, pp. 696-702, 2001

[24] S.M. Singh and K. Hemachandran, Content-Based Image Retrieval using Color Moment and Gabor Texture Feature, Int. J. of Comput. Sci. Issues, vol.9, no.1, pp. 299-309, 2012

[25] E. Tuncel, P. koulgi and K. Rose, Rate-distortion approach to databases: storage and content-based retrieval, IEEE Trans. Inf. Theory, vol.50, no.6, pp. 953-967, 2004

[26] W. Luo, H. Li, G. Liu and L. Zeng, Semantic Annotation of Satellite Images Using Author-Genre-Topic Model, IEEE Trans. on Geos. Rem. Sens., vol. 52, no. 2, pp. 1356-1368, 2014

[27] W. Luo, H. Li and G. Liu, Automatic Annotation of Multispectral Satellite Images Using Author-Topic Model, IEEE Geos. Rem. Sens. Lett., vol. 9, no. 4, pp. 634-638, 2012

[28] J. Wu and M. R. James, CENTRIST: A visual descriptor for scene categorization, IEEE Trans. Pattern Anal. Mach. Intell., vol. 33, no. 8, pp. 1489-1501, 2011

[29] Y. Hongyu, L. Bicheng and C. Wen, Remote sensing imagery retrieval based-on Gabor texture feature classification, Proc. IEEE Signal Process., vol.1, pp. 733-736, 2004 